\title{
Waveform Preconditioning for Clutter Rejection in Multipath for Sparse Distributed Apertures
}

\author{
T. Varslot, ${ }^{a}$ B. Yazıcı, ${ }^{a}$ C.-E Yarman, ${ }^{a}$ M. Cheney ${ }^{a}$ and L. Scharf. ${ }^{b}$ \\ ${ }^{a}$ Rensselaer Polytechnic Institute, Troy, NY, USA \\ ${ }^{b}$ Colorado State University, Fort Collins, CO, USA
}

\begin{abstract}
The idea of preconditioning transmit waveforms for optimal clutter rejection in radar imaging is presented. Waveform preconditioning involves determining a map on the space of transmit waveforms, and then applying this map to the waveforms before transmission. The work applies to systems with an arbitrary number of transmitand receive-antenna elements, and makes no assumptions about the elements being co-located. Waveform preconditioning for clutter rejection achieves efficient use of power and computational resources by distributing power properly over a frequency band and by eliminating clutter filtering in receive processing.
\end{abstract}

Keywords: Clutter rejection, waveform preconditioning, distributed aperture, sparse aperture, waveform design, imaging.

\section{INTRODUCTION}

In this paper we show how we by appropriate filtering of the transmit signals can design waveforms that suppress undesired features in the scattered signal. This filter will be referred to as a preconditioning operator; it is applied to the transmit waveform prior to transmission. In radar applications the scene (everything in the radar beam) is composed of three classes: objects of interest, objects which are not of interest, and (known) background. Objects of interest are referred to as targets, while those objects which are not of interest are referred to as clutter. In this paper, scattering from clutter is an undesired feature to be rejected by means of a waveform preconditioning operator. Clutter rejection is an important task, as scattering from clutter can overpower scattering from targets, thus rendering the targets difficult to detect or image. Our primary application is radar imaging. It should be clear, however, that our physics-based approach, which we formulate in terms of Green's functions and secondorder random fields, is applicable to pulse-echo imaging in general, e.g. ultrasound imaging, sonar imaging and microwave imaging.

There are two main waveform design approaches in the radar literature, namely the ambiguity and variationalbased approaches. In the first approach, a range-Doppler echo model is used with matched-filter processing. The waveforms are designed and combined in order to create an approximate Dirac-delta ambiguity function. ${ }^{1-10}$ In the second approach, the scene is assumed to be static and therefore the range-only echo model is considered. Similar to the first approach, matched filtering is used as a foundation for joint design of waveforms and receive processing for target detection, identification and classification. ${ }^{11-15}$ For target detection, the waveforms are designed by maximizing the signal-to-noise or signal-to-interference ratios. ${ }^{12-14} \mathrm{~A}$ mutual information criteria has also been used to design optimal waveforms for target classification. ${ }^{12}$ In both of the aforementioned approaches a monostatic radar system is considered.

Optimal recovery of a deterministic distribution of targets was presented by Naparst. ${ }^{16}$ Recently, Yazici et al. introduced waveform preconditioning as an approach to waveform design under the assumption that an $a$ priori stochastic characterization of the scattering distribution is available. ${ }^{17}$

\footnotetext{
Further author information: (Send correspondence to T. Varslot)

T. Varslot: e-mail: trond@ecse.rpi.edu, telephone: +1 (518) 2766357

B. Yazıcı: e-mail: yazici@ecse.rpi.edu, telephone: +1 (518) 2762905

M. Cheney: e-mail: cheney@rpi.edu, telephone: +1 (518) 2762646

L Scharf: $\quad$ email: scharf@engr.colostate.edu, telephone: +1 (970) 491-2979
}

Algorithms for Synthetic Aperture Radar Imagery XIV, edited by Edmund G. Zelnio, Frederick D. Garber, Proc. of SPIE Vol. 6568, 65680N, (2007) · 0277-786X/07/\$18 - doi: 10.1117/12.719570 
There is also a growing interest in combining multiple radar antennas to form distributed radar systems. This require special attention since the same waveform need not be transmitted from all antennas. The concept of Multiple-Input-Multiple-Output (MIMO) radar has been used to explore the advantages of multiple antennas. ${ }^{18,19}$

We formulate the processing of radar data from an arbitrary number of transmit- and receive-antennas in a stochastic framework. In this sense our work is closely related to Yazici et al. ${ }^{17}$ where an a priori stochastic characterization of the scattering distribution was used to construct optimal waveforms for mono-static rangeDoppler imaging. It should be clear, however, that our idea also applies to other situations such as suppression of multipath artifacts, enhancement of features for target identification.

From a communications point of view, the radar transmit signal which illuminates the target may be considered as a means for establishing a communications channel between the target and the observer. In this language, the effect of a complex environment is considered as part of this communications channel. The goal is to design a filter which, when applied to the transmit signal, virtually shapes the received signal in a desired manner. Thus, there are obvious similarities between the ideas presented here for radar imaging, and the existing literature on precoding. ${ }^{20-22}$ However, since imaging applications primarily aim at reconstructing the spatial distribution of the target, the spatial aspects of the wave propagation are pronounced in our work.

\section{PRELIMINARY THEORY AND MODELING}

We will consider a radar antenna consisting of $m$ transmitting elements, and $n$ receiving elements. The placement of these elements can be chosen arbitrarily, i.e., the location of each element may be assigned independently of where the other elements are located. For simplicity, we assume each element to behave like an isotropic point antenna, i.e., radiation patterns from each element do not exhibit any directivity. The latter assumption is not needed, but is made in order to make the analysis more transparent. An important feature of the system under consideration is that we have a reference clock which is common to all of the elements. This common reference clock facilitates coherent data processing, i.e., full knowledge of the signal phase as well as amplitude. An illustration of the distributed nature of an antenna with two transmitting elements and ten receiving elements is shown in Fig. 1.

In order to exploit the spatial diversity inherent in the antenna, it is desirable to allow for transmitting different waveforms from each element. Let $s_{j}(t)$ denote the waveform which emanates from the $j^{\text {th }}$ element. The transmit waveforms are arranged in a transmit vector $\boldsymbol{s}(t)$

$$
\boldsymbol{s}(t)=\left[s_{1}(t), \ldots, s_{m}(t)\right]^{\mathrm{T}} .
$$

Similarly, if the measured scattering at the $i^{\text {th }}$ receive element is denoted by $m_{i}(t)$, then the scattering which is collected by the distributed antenna may be arranged in a measurement vector $\boldsymbol{m}(t)$

$$
\boldsymbol{m}(t)=\left[m_{1}(t), \ldots, m_{n}(t)\right]^{\mathrm{T}} .
$$

\subsection{Scattering model}

The ability to distinguish targets depends on how much their electromagnetic properties deviate from a known background. We denote this deviation by the scattering potential $V(\boldsymbol{x})$. At an abstract level, we denote by $\mathcal{H}(V)$ the operator which maps the transmit vector $\boldsymbol{s}(t)$ to the measurement vector $\boldsymbol{m}(t)$

$$
\boldsymbol{m}(t)=\mathcal{H}(V) \boldsymbol{s}(t) .
$$

An explicit relationship between the scattering potential $V$ and the operator $\mathcal{H}(V)$ can be derived in terms of the Green's function $g(\boldsymbol{x}, \boldsymbol{y}, t)$ for the background medium. The Green's function is the response measured at position $\boldsymbol{x}$ from an impulse $\delta(t)$ at position $\boldsymbol{y}$. The geometric layout of the antenna elements naturally plays an important role here. Let therefore the $j^{\text {th }}$ transmit element be located at position $\boldsymbol{z}_{j}$, and the $i^{\text {th }}$ receive 


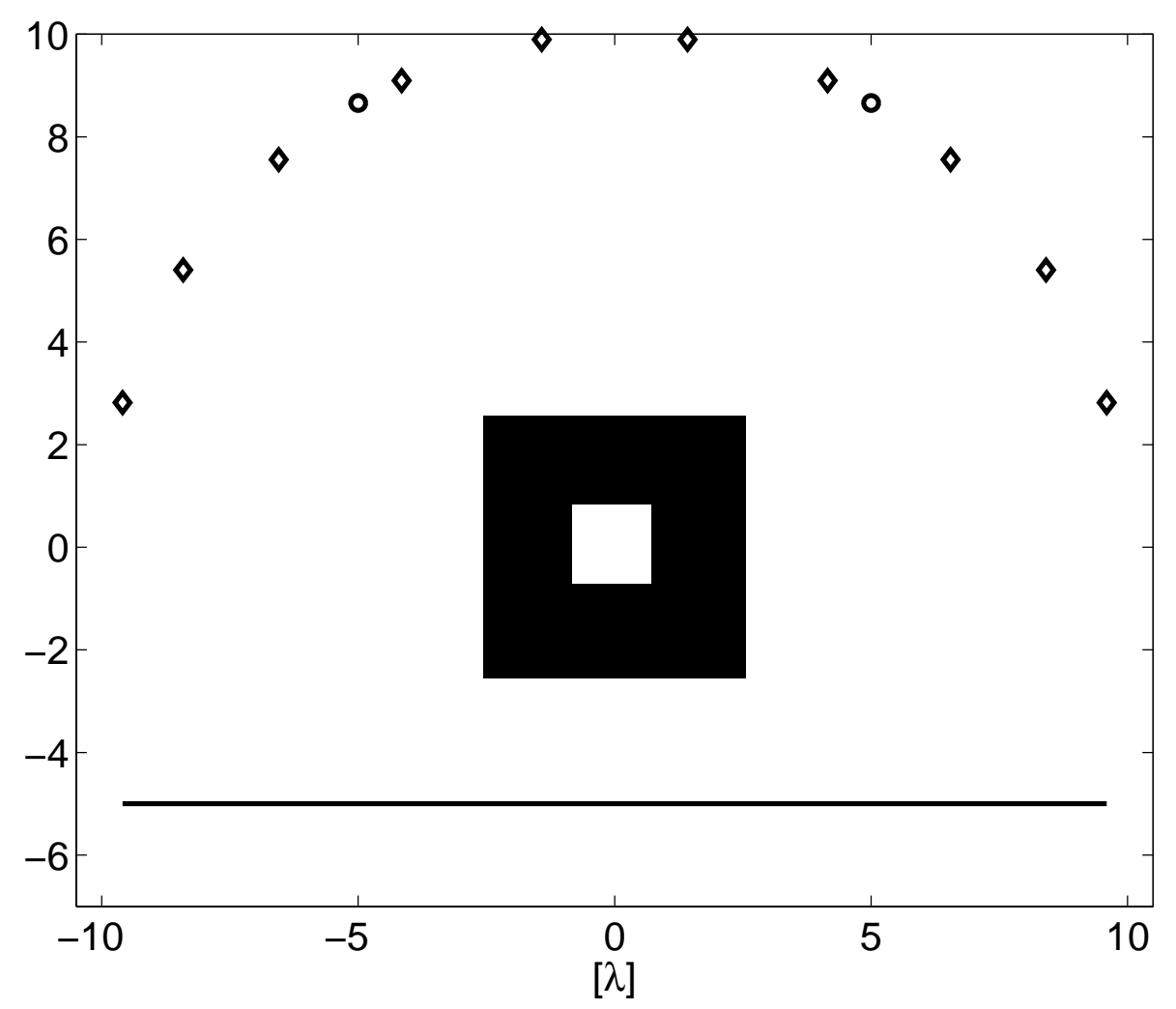

Figure 1. Distributed radar antenna with two transmitting elements (circles) and ten receiving elements (diamonds). The elements are placed at equidistant points along a an arc with radius $10 \lambda$, where $\lambda$ is the wavelength corresponding to the center frequency of the transmitters. The target is indicated as a square with sides of $1.5 \lambda$, while the region of interest is $5 \lambda \times 5 \lambda$ around the target. This region is filled with clutter. The solid straight line indicates the location of a reflective mirror in our simple multipath scenario

element be located at position $\boldsymbol{x}_{i}$. In the current analysis we use a linear scattering model often known as the distorted-wave Born approximation. ${ }^{23}$ If we define a $(m \times n)$ matrix $G(\boldsymbol{y}, t)$ with matrix elements

$$
G_{i j}(\boldsymbol{y}, t)=\int g\left(\boldsymbol{z}_{j}, \boldsymbol{y}, \tau^{\prime}\right) \partial_{t}^{2} g\left(\boldsymbol{y}, \boldsymbol{x}_{i}, t-\tau^{\prime}\right) \mathrm{d} \tau^{\prime},
$$

then

$$
\boldsymbol{m}(t)=\mathcal{H}(V) \boldsymbol{s}(t)=\int G(\boldsymbol{y}, t-\tau) V(y) \mathrm{d} \boldsymbol{y} \boldsymbol{s}(\tau) \mathrm{d} \tau .
$$

Integration in Eq. (5) is understood to be element-wise.

The map $\mathcal{H}(V)$ is linear from the space of transmit vectors to the space of measurement vectors. By employing a set of transmit waveform vectors $\left\{\boldsymbol{s}_{k}(t)\right\}$, the action of $\mathcal{H}(V)$ can be determined on the space spanned by these vectors. Once the map is known, imaging is formally performed as

$$
\hat{V}(\boldsymbol{x})=\mathcal{H}^{-1} \mathcal{H}(V),
$$

where $\mathcal{H}^{-1}$ is an approximate inverse that can be computed using the prior knowledge about the background. ${ }^{24}$ Here we will not discuss further how we can perform this inversion, but rather focus our attention to clutter rejection. 


\subsection{Target and clutter}

The above formalism allows us to utilize a physics-based model for the background which in principle can have an arbitrary level of detail. It is not reasonable, however, to expect that our background model will account for all details of the scene except for the target. As outlined in the introduction, this suggests that the scattering potential should be divided into two parts

$$
V(\boldsymbol{x})=T(\boldsymbol{x})+C(\boldsymbol{x}),
$$

where $T(\boldsymbol{x})$ represents target and $C(\boldsymbol{x})$ represents clutter. By this approach, our definition of clutter includes contrubutions due to a compromise between model fidelity and tractability: deviations between the background and our model for the background. The real interest lies in recovering $T$, while suppressing $C$.

In many situations it is reasonable to assume that information about statistics of the scattering is available. There is a large body of literature on stochastic signal models for electromagnetic waves (see e.g., Sarkar et al. ${ }^{25}$ and the references therein). Here we have chosen to impose a stochastic model for the scattering potential itself.

In our development we assume that $T(\boldsymbol{x})$ and $C(\boldsymbol{x})$ are realizations of second-order random fields with known first- and second-order statistics. It should be clear that the background may be defined in such a way that the first-order statistics of the scattering potentials are zero. Thus, without loss of generality, we will assume that the processes have zero-mean and known auto-correlation functions

$$
\begin{aligned}
& R_{T}\left(\boldsymbol{y}_{1}, \boldsymbol{y}_{2}\right)=\mathrm{E}\left[T\left(\boldsymbol{y}_{1}\right) \overline{T\left(\boldsymbol{y}_{2}\right)}\right] \\
& R_{C}\left(\boldsymbol{y}_{1}, \boldsymbol{y}_{2}\right)=\mathrm{E}\left[C\left(\boldsymbol{y}_{1}\right) \overline{C\left(\boldsymbol{y}_{2}\right)}\right] .
\end{aligned}
$$

Finally, we will assume that the fields $T$ and $C$ are statistically independent, i.e.,

$$
\begin{aligned}
R_{V}\left(\boldsymbol{y}_{1}, \boldsymbol{y}_{2}\right) & =\mathrm{E}\left[\left(T\left(\boldsymbol{y}_{1}\right)+C\left(\boldsymbol{y}_{1}\right) \overline{\left(T\left(\boldsymbol{y}_{2}\right)+C\left(\boldsymbol{y}_{2}\right)\right)}\right]\right. \\
& =R_{T}\left(\boldsymbol{y}_{1}, \boldsymbol{y}_{2}\right)+R_{C}\left(\boldsymbol{y}_{1}, \boldsymbol{y}_{2}\right) .
\end{aligned}
$$

The fact that the scattering potential is stochastic will of course imply that the kernel of the integral operator in Eq. (5) is stochastic [26, Ch. 2]. Furthermore, it directly relates the statistics of the electromagnetic scattering received by a radar antenna to the statistics of the scattering potential. Thus, we assume that Eq. (5) is defined in the mean-square sense.

We will suppress $C$ by modifying the transmit waveforms such that they produce mostly scattering from $T$. We formulate this as determining an operator $\mathcal{W}$ such that

$$
\mathcal{H}(T+C) \mathcal{W} \approx \mathcal{H}(T)
$$

We achieve clutter rejection by employing the transmit waveform $\mathcal{W} s$ instead of $s(t)$. Our approach is illustrated in Fig. 2.

In this work we choose to reject clutter in the minimum-mean-square-error (MMSE) sense. To be more specific: under mild assumptions about the transmit waveforms and the scattering potentials we can show that $\mathcal{H}(V)$ is a Hilbert-Schmidt (HS) operator [27, Ch. 6.2]. Our goal is therefore to determine a linear operator $\mathcal{W}$ which minimizes the HS norm

$$
\mathcal{W}=\underset{\mathcal{W}}{\operatorname{argmin}}\|\mathcal{H}(T+C) \mathcal{W}-\mathcal{H}(T)\|_{\mathrm{HS}}^{2} .
$$

Recall that the HS norm is induced by the inner product between HS operators $A$ and $B$ defined as follows

$$
\langle A, B\rangle_{\mathrm{HS}} \equiv \sum_{k}\left\langle A \boldsymbol{e}_{k}, B \boldsymbol{e}_{k}\right\rangle \equiv \operatorname{Tr}\left\{B^{*} A\right\},
$$




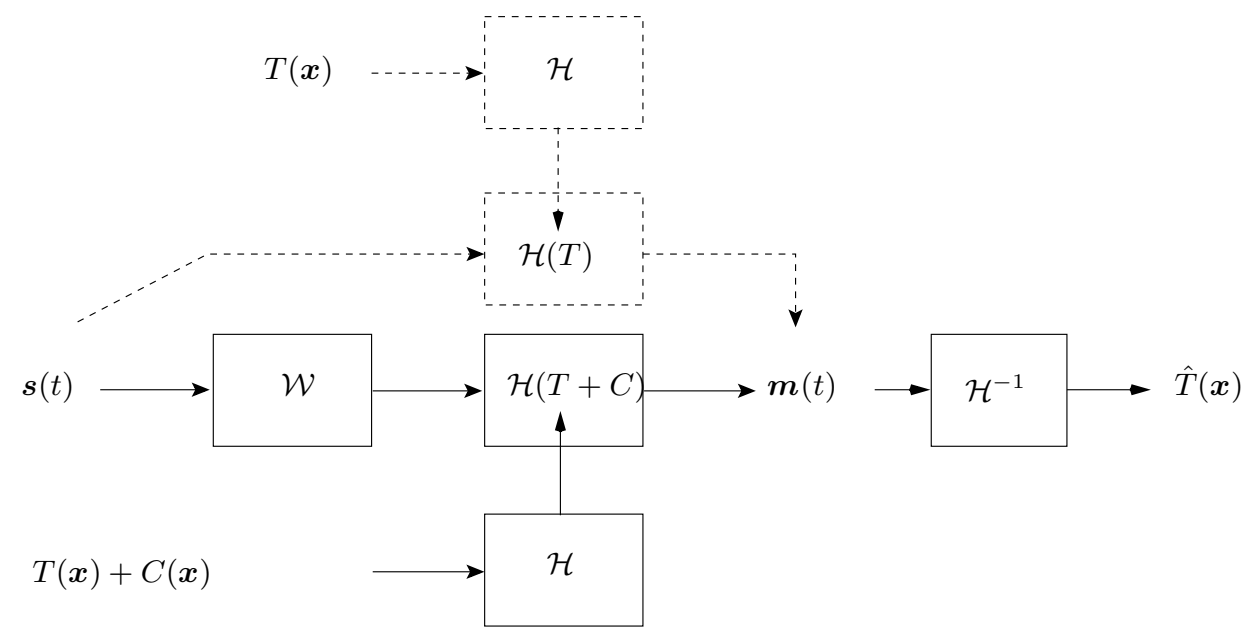

Figure 2. Block diagram for the radar imaging process with waveform preconditioning operator $\mathcal{W}$. Solid arrows indicate the signal path from waveform, through preconditioning operator and then transmitted into an environment with system response $\mathcal{H}(T+C)$. Dashed lines indicate the "ideal" signal path which is emulated by applying the preconditioning operator.

where $\left\{\boldsymbol{e}_{k}\right\}$ is a orthonormal complete basis for the domain of the operators, $B^{*}$ is the adjoint of the operator $B$, and $\operatorname{Tr}\{\cdot\}$ denotes the trace. Since we are dealing with stochastic signals, we employ the following inner product between random vector processes in Eq. $14^{28}$

$$
\langle\boldsymbol{x}, \boldsymbol{y}\rangle=\sum_{i} \int \mathrm{E}\left[x_{i}(t) \overline{y_{i}(t)}\right] \mathrm{d} t
$$

We observe that the HS norm is simply the sum of $L^{2}$ norms for an orthonormal basis of transmit vectors. Hence, determining $\mathcal{W}$ according to Eq. (13) will in our stochastic setting minimize the average MMSE over these orthonormal transmit vectors.

\section{THE PRECONDITIONING OPERATOR}

In this section we will derive an explicit expression for $\mathcal{W}$ as defined in Eq. (13). In order for the analysis to hold, it is necessary to make certain assumptions about the transmit waveforms and the scattering environment. These assumptions are mild enough to be satisfied for any practical application.

First of all, we need to consider only transmit waveforms which have finite length, and which has sufficient spectral decay as a function of frequency. For a transmit waveform $s(t)$ with Fourier transform $\hat{s}(\omega)$, this asymptotic decay can be made precise by requiring that ${ }^{24}$

$$
\int\left(1+\omega^{6}\right)|\hat{s}(\omega)|^{2} \mathrm{~d} \omega<\infty
$$

Second it is convenient to assume that no echoes emerge from outside a sufficiently large region, i.e., that the scattering potential has compact support. In a practical situation where additive noise is present it is not reasonable to expect scattering to be detectable from arbitrary large distances.

Finally we will assume that the correlation function $R_{V}\left(\boldsymbol{y}_{1}, \boldsymbol{y}_{2}\right)$ is absolutely integrable

$$
\int\left|R\left(\boldsymbol{y}_{1}, \boldsymbol{y}_{2}\right)\right| \mathrm{d} \boldsymbol{y}_{1} \mathrm{~d} \boldsymbol{y}_{2}<\infty .
$$


Under the aforementioned assumptions about the waveforms and scattering environment, we can show that $\mathcal{H}(V)$ is a HS operator. We are therefore justified in using the HS norm when determining the preconditioning operator.

Let $\left\{\boldsymbol{e}_{k}(t)\right\}$ be a basis for the space of transmit vectors. By combining Eqns. (14) and (15) we see that the HS norm of $\mathcal{H}(V)$ can be computed as

$$
\|\mathcal{H}(V)\|_{\mathrm{HS}}^{2}=\sum_{k} \int \boldsymbol{e}_{k}^{\mathrm{H}}(t) \mathrm{E}\left[\mathcal{H}(V)^{*} \mathcal{H}(V)\right] \boldsymbol{e}_{k}(t) \mathrm{d} t,
$$

where

$$
\mathrm{E}\left[\mathcal{H}(V)^{*} \mathcal{H}(V)\right] \boldsymbol{e}_{k}(t)=\mathcal{G}^{*} \mathcal{R}_{V} \mathcal{G} \boldsymbol{e}_{k}(t) .
$$

Here $*$ denotes the adjoint, and $\mathrm{H}$ denotes the conjugate transpose of a vector. Furthermore, $\mathcal{G}$ is an integral operator with (matrix) kernel $G(\boldsymbol{y}, t, \tau)$, and $\mathcal{R}_{V}$ is an integral operator with (scalar) kernel $R_{V}\left(\boldsymbol{y}_{1}, \boldsymbol{y}_{2}\right)$, i.e.,

$$
\begin{aligned}
\mathcal{G} \boldsymbol{f}(\boldsymbol{y}, t) & =\int G(\boldsymbol{y}, t, \tau) \boldsymbol{f}(\tau) \mathrm{d} \tau, \\
\mathcal{R}_{V} \boldsymbol{f}\left(\boldsymbol{y}_{1}\right) & =\int R_{V}\left(\boldsymbol{y}_{1}, \boldsymbol{y}_{2}\right) \boldsymbol{f}\left(\boldsymbol{y}_{2}\right) \mathrm{d} \boldsymbol{y}_{2} .
\end{aligned}
$$

We now determine the operator $\mathcal{W}$ which minimizes

$$
\Delta(\mathcal{W}) \equiv\|\mathcal{H}(T+C) \mathcal{W}-\mathcal{H}(T)\|_{\mathrm{HS}}^{2}
$$

This minimum is found by means of variational analysis. If we require that

$$
\Delta(\mathcal{W}+\delta \mathcal{W})-\Delta(\mathcal{W})=\mathcal{O}\left(\|\delta \mathcal{W}\|^{2}\right)
$$

for all perturbations $\delta \mathcal{W}$, we obtain the following

$$
\begin{aligned}
0 & =\langle\mathcal{H}(T+C) \delta \mathcal{W},[\mathcal{H}(T+C) \mathcal{W}-\mathcal{H}(T)]\rangle_{\mathrm{HS}} \\
& =\operatorname{Tr}\left\{\mathrm{E}\left[(\mathcal{H}(T+C) \mathcal{W}-\mathcal{H}(T))^{*} \mathcal{H}(T+C)\right] \delta \mathcal{W}\right\}
\end{aligned}
$$

We then evaluate the expectations in terms of deterministic functions, in a similar fashion to what was performed in Eq. (19). Since $T$ and $C$ are stochastically independent

$$
\begin{aligned}
\mathrm{E}\left[(\mathcal{H}(T+C) \mathcal{W})^{*} \mathcal{H}(T+C)\right] & =\mathcal{W}^{*} \mathcal{G}^{*}\left(\mathcal{R}_{T}+\mathcal{R}_{C}\right) \mathcal{G} \\
\mathrm{E}\left[(\mathcal{H}(T))^{*} \mathcal{H}(T+C)\right] & =\mathcal{G}^{*} \mathcal{R}_{T} \mathcal{G}
\end{aligned}
$$

The kernels of the operators $\mathcal{R}_{T}$ and $\mathcal{R}_{C}$ are the auto-correlation functions for $T$ and $C$, respectively. In order for Eq. (25) to hold for all $\delta \mathcal{W}$, all basis vectors must be in the null-space of

$$
\mathcal{G}^{*}\left(\mathcal{R}_{T}+\mathcal{R}_{C}\right) \mathcal{G} \mathcal{W}-\mathcal{G}^{*} \mathcal{R}_{T} \mathcal{G} .
$$

Hence we find that

$$
\mathcal{W}=\left(\mathcal{G}^{*}\left[\mathcal{R}_{T}+\mathcal{R}_{C}\right] \mathcal{G}\right)^{\dagger} \mathcal{G}^{*} \mathcal{R}_{T} \mathcal{G}
$$

Here $\dagger$ denotes pseudo-inverse.

Both $\mathcal{G}^{*} \mathcal{R}_{T} \mathcal{G}$ and $\mathcal{G}^{*}\left[\mathcal{R}_{T}+\mathcal{R}_{C}\right] \mathcal{G}$ are compact self-adjoint operators. Therefore, they have a spectral representation in the form of a discrete sum of eigenfunctions. ${ }^{29}$ Let $\left\{\left(\lambda_{k}, \boldsymbol{u}_{k}\right)\right\}$ be a set of eigenvalues/eigenfunctions for $\mathcal{G}^{*} \mathcal{R}_{T} \mathcal{G}$, and $\left\{\left(\sigma_{k}, \boldsymbol{v}_{k}\right)\right\}$ be a set of eigenvalues/eigenfunctions for $\mathcal{G}^{*}\left(\mathcal{R}_{T}+\mathcal{R}_{C}\right) \mathcal{G}$

$$
\begin{aligned}
\lambda_{k} \boldsymbol{u}_{k} & =\mathcal{G}^{*} \mathcal{R}_{T} \mathcal{G} \boldsymbol{u}_{k} \\
\sigma_{k} \boldsymbol{v}_{k} & =\mathcal{G}^{*}\left(\mathcal{R}_{T}+\mathcal{R}_{C}\right) \mathcal{G} \boldsymbol{v}_{k} .
\end{aligned}
$$


Then $\mathcal{W}$ can be written as

$$
\begin{aligned}
\mathcal{W} s & =\sum_{l} \frac{1}{\sigma_{l}}\left\langle\sum_{k} \lambda_{k}\left\langle\boldsymbol{s}, \boldsymbol{u}_{k}\right\rangle \boldsymbol{u}_{k}, \boldsymbol{v}_{l}\right\rangle \boldsymbol{v}_{l} \\
& =\sum_{l} \sum_{k} \frac{\lambda_{k}}{\sigma_{l}}\left\langle\boldsymbol{s}, \boldsymbol{u}_{k}\right\rangle\left\langle\boldsymbol{u}_{k}, \boldsymbol{v}_{l}\right\rangle \boldsymbol{v}_{l} .
\end{aligned}
$$

This operator may be applied to any transmit vector to yield a new transmit vector; $\mathcal{W}$ is a bounded linear operator on the space of transmit vectors. Loosely speaking, $\mathcal{W}$ emphasizes the parts of $s$ residing the subspaces in which the signal-to-clutter ratio is relatively high.

\section{NUMERICAL SIMULATION}

In order to demonstrate the clutter-suppression obtained with our waveform preconditioning operator, we have performed a set of numerical simulations. In these simulations we want to recover the target $T$ from scattering measurements made with two transmitters and ten receivers. The transmitters and receivers were placed equally spaced on an arc around the target. This simulation setup is illustrated in Fig. 1.

We have performed two numerical experiments. First we simulated scattering from only the target and the clutter. Then we perform another simulation where we insert a mirror surface behind the target (see Fig. 1. The role of this mirror is to illustrate a simple multipath scenario.

From the two transmitters we transmitted short chirp signals: transmitter 1 emitted a linear up-chirp, while transmitter 2 simultaneously emanated a linear down-chirp. All dimensions of the experiment were normalized according to the wavelength corresponding to the center frequency. We denote this wavelength by $\lambda$.

As a target we chose a square with sides $1.5 \lambda \times 1.5 \lambda$. From this target model we constructed a target spectrum as if the target were a realization of a stationary random field. A high-frequency version of the stationary stochastic target model was then constructed and used to simulate different realizations of the surrounding clutter. This construction is explained further in Yazici et al. ${ }^{30}$ The compact support of the clutter was imposed by applying a spatial mask. For our purpose we used a region of $5 \lambda \times 5 \lambda$ around the target. Finally, the radius of the arc on which the antenna elements were placed was set to $10 \lambda$.

The preconditioning operator was constructed according to Eq.(29) by a Monte-Carlo approach where we used 500 realizations of the stochastic fields $T$ and $C$. The spatial discretization for each scattering simulation was 15 samples per wavelength $\lambda$.

The signal-to-clutter ratio (SCR) in our simulations was set to $-3.5 \mathrm{~dB}$, when defined according to

$$
\mathrm{SCR}=10 \log \left(\frac{\int \mathrm{E}\left[|T(\boldsymbol{x})|^{2}\right] \mathrm{d} \boldsymbol{x}}{\int \mathrm{E}\left[|C(\boldsymbol{x})|^{2}\right] \mathrm{d} \boldsymbol{x}}\right)
$$

The performance of the preconditioning was then evaluated by observing the square error in the reconstructed image when compared to the true scattering potential. The mean-square-error (MSE) was estimated by averaging over 10 clutter realizations. We computed the MSE according to

$$
\operatorname{MSE}(\mathcal{W})=10 \log \left(\frac{\int \mathrm{E}\left[\left|\left[\mathcal{H}^{-1} \mathcal{H}(T+C) \mathcal{W}\right](\boldsymbol{x})-T(\boldsymbol{x})\right|^{2}\right] \mathrm{d} \boldsymbol{x}}{\int \mathrm{E}\left[|T(\boldsymbol{x})|^{2}\right] \mathrm{d} \boldsymbol{x}}\right) .
$$

To be consistent with the MMSE-criterion from which the preconditioning was constructed, we used an imaging reconstruction algorithm which is based on minimising the MSE of the final image. ${ }^{24}$ The images were reconstructed on a grid with 10 samples per wavelength.

Figure 3 shows the target embedded in clutter, as well as a reconstructed image based on scattering without clutter in free space. The MSE is in this case was $-3.7 \mathrm{~dB}$.

Figures 4 and 5 show reconstruction results for scattering with clutter in free space and in multipath scenarios, respectively. For the free space scenario, preconditioning of the transmit waveform improves image quality from 

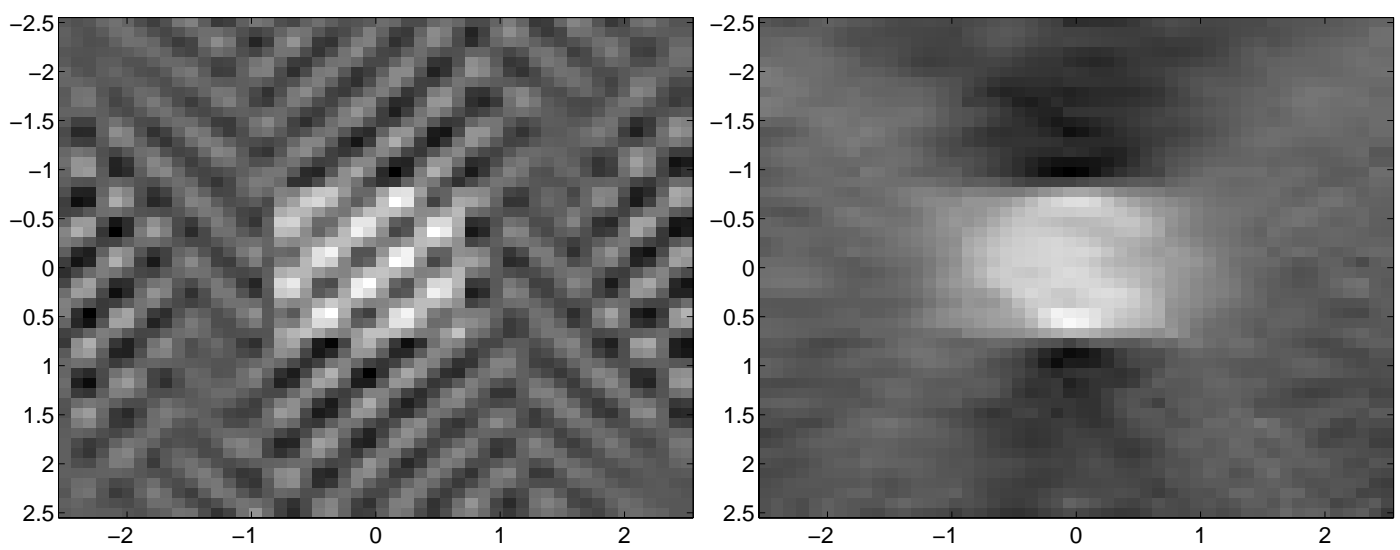

Figure 3. Target with clutter (left) and reconstruction of target from clutter-free scattering (right)
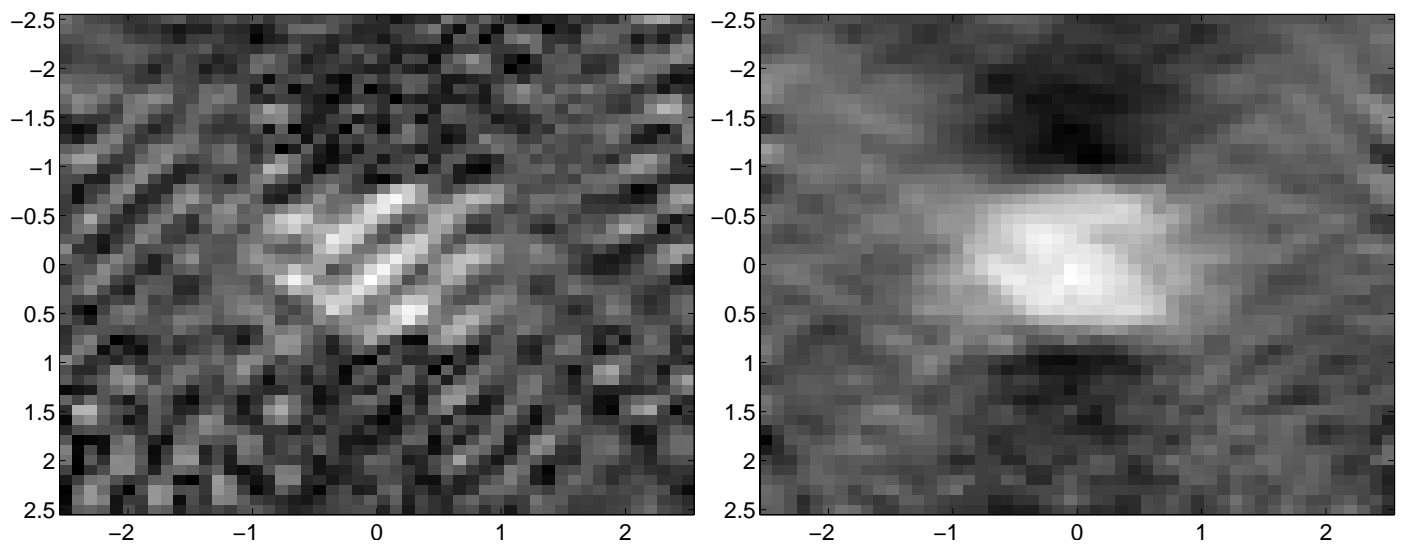

Figure 4. Reconstruction results for a single realization of clutter in free space simulation. Left: image from scattering with original chirp waveform. Right: image from scattering with preconditioned chirp waveform
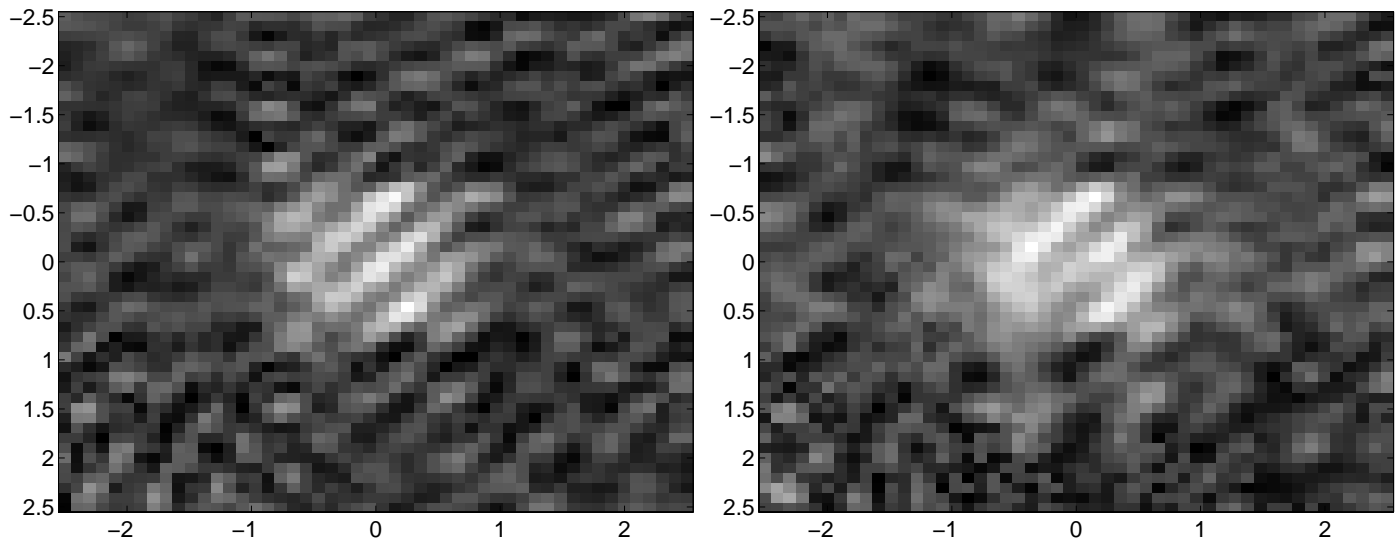

Figure 5. Reconstruction results for a single realization of clutter in multipath simulation. Left: image from scattering with original chirp waveform. Right: image from scattering with preconditioned chirp waveform. 

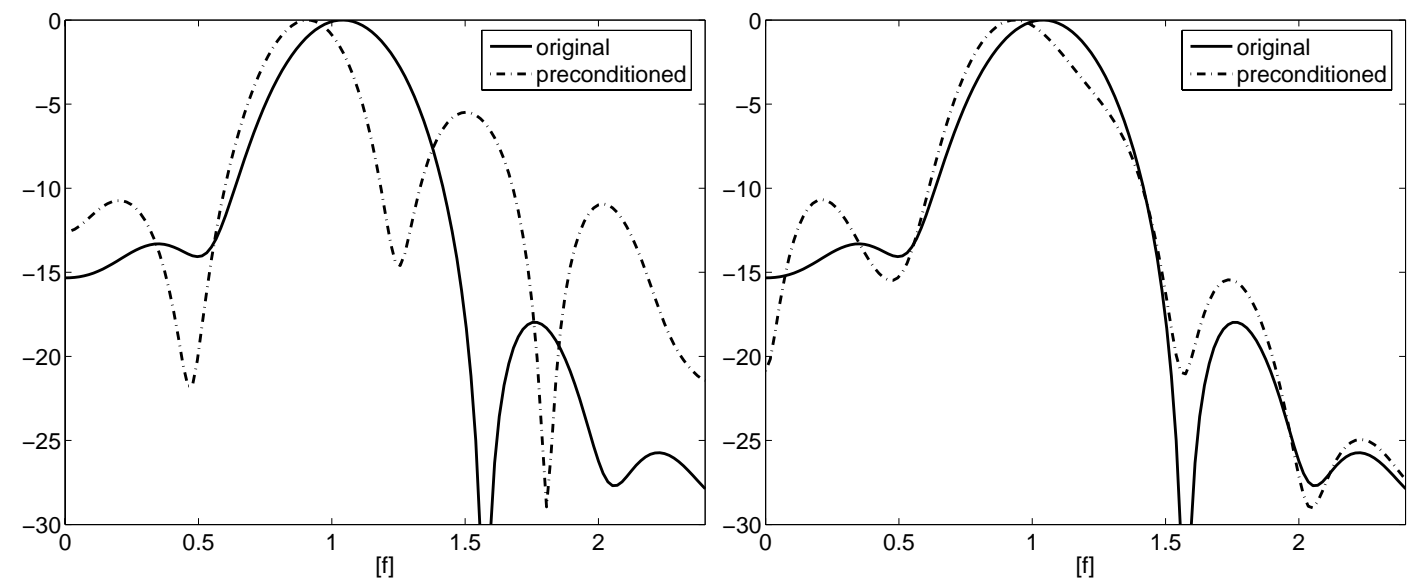

Figure 6. Spectrum of transmit waveforms. Left: preconditioned for clutter rejection in free space. Right: preconditioned for clutter rejection in multipath environment.

$1.1 \mathrm{~dB}$ to $-3.6 \mathrm{~dB}$ when measured using the MSE defined in Eq. (35). For the multipath situation, however, preconditioning improves the result from $0.1 \mathrm{~dB}$ to $-1.4 \mathrm{~dB}$.

Figure 6 shows the spectrum of one of the transmit waveform that were employed in this experiment. We note that the most dramatic modification of the waveform occurs for the free space scenario.

\section{DISCUSSION AND CONCLUDING REMARKS}

In this work we have separated the scattering potential into two distinct classes: target and clutter. The clutter essentially produces unwanted scattering which in turn degrades the final result of the reconstructed image. If scattering from clutter can be removed from the measurement, the end result will be improved. We have presented a method for preconditioning the transmit waveform in order to obtain this clutter rejection based on a priori information about the statistics of the scattering potential.

We address the problem of optimally modifying the transmit waveform when the transmit power is limited. Obviously, after preconditioning the resulting waveform may not have the same strength. However, this is trivially amended by proper normalization of the preconditioned waveform. As a result, the transmit vector will contain the same amount of power, but will minimize scattering from clutter.

When we perform clutter rejection we identify a transmit-vector subspace where the signal-to-clutter (SCR) ratio is high. The fact that the signals which are employed at each transmitter are transmitted simultaneously, and that they are not orthogonal imply that there is a great deal of ambiguity in the data with respect to the correct time-of-flight for a given echo. We therefore have limited ability to determine the correct source-reciever pair for a given echo. As we are forming the images using a our limited-angle tomographic approach, we to not rely on the ability to resolve the source-reciever ambiguity. ${ }^{24}$

Multipath may be modeled by adding more transmitters. This will increase the complexity of the propagation path and hence result in a more complex illumination pattern as compared to the free space scenario. However, our ability to control the illumination pattern does not increase as we are still restricted to use the same number of independently controllable transmit elements. Furthermore, by effectively illuminating the target and clutter from additional directions, it is harder to find a subspace where the SCR is high. One way to increase the flexibility would be to employ multiple transmit vectors, i.e., span a larger transmit vector space. Our preconditioning operator can be applied to any set of transmit vectors in order to oprimally reject scattering from clutter in the MMSE sense.

An important feature of the preconditioning approach is the improved SCR of the scattering. Hence, for a fixed total transmit power, the SCR may be improved in the final image. Alternatively, for a given signal-to-noise 
ratio in the final image the total transmit power can be reduced. This is of interest in applications where it is desirable to keep the transmit power as low as possible, e.g., to reduce transmitter vulnerability/detectability.

Our approach employs information about the scene in the form of the Green's function. This Green's function is then used to map second-order statistics of the clutter and target to the space of transmit vectors and thereby construct the preconditioning operator. The second-order statistics can be derived for each typical location; for example, different landscape types or housing styles yield different kinds of clutter statistics.

In Eq. (33) the preconditioning operator is expressed in terms of ratios between eigenvalues of $\mathcal{G}^{*} \mathcal{R}_{T} \mathcal{G}$ and $\mathcal{G}^{*}\left(\mathcal{R}_{T}+\mathcal{R}_{C}\right) \mathcal{G}$. It is therefore evident that the preconditioning operator will emphasize parts of the waveform which resides in subspaces which produces more scattering from target than from clutter. Our preconditioning can therefore be viewed as a subspace technique for transmit waveforms.

The underlying propagation model which we have used for this work is derived from a scalar wave equation. This is a commonly-used model for many radar applications where polarization effects may be ignored. In order to get explicit expressions in terms of Green's functions, a linearized scattering model was used, namely the distorted-wave Born approximation (DWB). Note, however, that the operator norm which we used to determined the preconditioning operator will make sense also without the DWB. A propagation model based on a scalar Green's function is applicable in many other applications such as ultrasound, sonar and microwave imaging. In situations where polarization is important, a similar framework can be developed using a dyadic Green's function.

Clutter filtering is an integral part of space-time adaptive processing (STAP). In STAP the statistics of the clutter is used to perform filtering of the measurement in order to reduce the clutter content. ${ }^{31}$ Filtering of the measurement implies that parts of the scattered power will not contribute to the final result. In this sense it is wasted scattering power. We obtain a similar result by filtering the transmit signal, thereby avoiding transmitting power which will be predominantly used to produce scattering from clutter. In this sense, the preconditioned transmit vector will yield more efficient use of the transmitters. Furthermore, by avoiding clutter filtering of the measurements, we reduce the computational resources needed at each the receiver element. Our scheme is therefore suited for applications where inexpensive receiver elements with limited computing power are employed, such as distributed sensing in urban environments.

The development in this paper was performed using the minimum-mean-square-error (MMSE) to define the optimal clutter rejection operator. This is a suitable in many applications. However, the idea can be extended to include other figures of merit, such as limited transmit power, limited bandwidth or absolute error. Although other figures of merit will change the optimal preconditioning operator, the same idea of preconditioning the transmit vectors remains can be used.

\section{ACKNOWLEDGMENTS}

We are grateful to Air Force Office of Scientific Research* (AFOSR) and the Defense Advanced Research Projects Agency (DARPA) for supporting this work under the agreements FA9550-04-1-0223, FA9550-06-1-0017 and FA8750-05-2-0285.

\section{REFERENCES}

1. R. H. Barker, Group synchronizing of binary digital systems, pp. 273-287. Academic Press, New York, 1953.

2. C. Wilcox, The synthesis problem for radar ambiguity functions, pp. 229-260. Springer-Verlag, 1991.

3. R. L. Frank, "Polyphase codes with good nonperiodic correlation properties," IEEE Trans. Inf. Theory 9(1), pp. 43-45, 1963.

4. R. Sivaswamy, "Digital and analog subcomplementary sequences for pulse compression," IEEE Trans. Aerosp. Electron. Syst. AES-14, pp. 343-350, 1978.

5. R. Sivaswamy, "Multiphase complementary codes," IEEE Trans. Inf. Theory IT-24(5), pp. 546-552, 1978.

\footnotetext{
${ }^{*}$ Consequently, the US Government is authorized to reproduce and distribute reprints for governmental purposes notwithstanding any copyright notation thereon. The views and conclusions contained herein are those of the authors and should not be interpreted as necessarily representing the official policies or endorsements, either expressed or implied, of the Air Force Research Laboratory or the US Government.
} 
6. J. P. Costas, "A study of a class of detection waveforms having nearly ideal range-doppler ambiguity properties," Proc. IEEE 72(8), pp. 996-1009, 1984.

7. S. W. Golomb and H. Taylor, "Constructions and properties of costas arrays," Proc. IEEE 72(9), pp. 11431163, 1984.

8. S. W. Golomb and H. Taylor, "Algebraic construction of costas arrays," J. Comb. Theory, ser. A 37, pp. 13-21, 1984.

9. J. Guey and M. Bell, "Diversity waveform sets for delay-doppler imaging," IEEE Transactions on Information Theory 44(4), pp. $1504-1522,1998$.

10. C. Chang and M. R. Bell, "Frequency-coded waveforms for enhanced delay-doppler resolution," IEEE Trans. Inf. Theory 49(11), pp. 2960-2971, 2003.

11. D. T. Gjessing, Target Adaptive Matched Illumination Radar: Principles and Applications, Peter Peregrinus, Stevenage, U.K., 1986.

12. M. Bell, "Information theory and radar waveform design," IEEE Trans. Inf. Theory 39, pp. 1578-1597, September 1993.

13. S. U. Pillai, H. S. Oh, D. C. Youla, and J. R. Guerci, "Optimum transmit-receiver design in the presence of signal-dependent noise and channel noise," IEEE Trans. Inf. Theory 46(2), pp. 577-584, 2000.

14. D. A. Garren, M. K. Osborn, A. C. Odom, J. S. Goldstein, S. U. Pillai, and J. R. Guerci, "Enhanced target detection and identification via optimized radar transmission pulse shape," Proc. IEE, Radar, Sonar and Navigation 148(3), pp. 130-138, 2001.

15. S. Sowelam and A. H. Tewfik, "Waveform selection in radar target classification," IEEE Transactions on Information Theory 46(3), pp. 1014-1029, 2000.

16. H. Naparst, "Dense target signal processing," IEEE Trans. Inform. Theory 37(2), pp. 317-327, 1991.

17. B. Yazıcı and G. Xie, "Wideband extended range-Doppler imaging and waveform design in the presence of clutter and noise," IEEE Trans. Inf. Theory 52(10), 2006.

18. E. Fishler, A. Haimovich, R. Blum, L. Cimini, D. Chizhik, and R. Valenzuela, "Spatial diversity in radars - models and detection performance," IEEE Trans. Sign. Processing 54(3), pp. 823-238, 2006.

19. I. Bekkerman and J. Tabrikian, "Target detection and localization using mimo radars and sonars," IEEE Tran. Signal Proc. 54(10), pp. 3873-3883, 2006.

20. G. Forney and M. Eyuboglu, "Combined equalization and coding using precoding," IEEE Comm Mag , Dec 1991.

21. H. Harashima and H. Miyakawa, "Matched transmission technique for channels with intersymbol interference," IEEE Trans. Commun. COM-20, pp. 774-780, 1972.

22. M. Tomlinson, "New automatic equalizer employing modulo arithmetic," Electron. Lett. 7, pp. 138-139, 1971.

23. J. H. Taylor, Scattering Theory, Wiley, New York, 1972.

24. T. Varslot, B. Yazıcı, and M. Cheney, "Pulse-echo imaging with distributed apertures in multi-path environments." (reprint), 2006.

25. T. K. Sarkar, Z. Ji, K. Kim, A. Medouri, and M. Salazar-Palma, "A survey of various propagation models for mobile communication," IEEE Ant. and Prop. Magazine 45, pp. 51-82, June 2003.

26. A. T. Bharucha-Reid, Random integral equations, vol. 96 of Mathematics in Science and Engineering, Academic Press, New York, 1972.

27. J. Weidmann, Linear Operators in Hilbert Spaces, Springer-Verlag, New York, 1980.

28. P. Todorovic, An introduction to stochastic processes and their applications, Statistics, Springer-Verlag, New York, 1992.

29. E. Kreyszig, Introductory Functional Analysis, Wiley, 1989.

30. B. Yazıcı, M. Cheney, and C. E. Yarman, "Synthetic aperture inversion for an arbitrary flight trajectory in the presence of noise and clutter," Inverse Problems 22(5), pp. 1705-1729, 2006.

31. J. Guerci, Space-Time Adaptive Processing, Artech House, Northwood, MA (USA), 2003. 